\title{
WHEN RATIONAL REASONERS REASON DIFFERENTLY
}

\author{
MICHAEL G. TITELBAUM AND MATTHEW KOPEC
}

\begin{abstract}
Different people reason differently, which means that sometimes they reach different conclusions from the same evidence. We maintain that this is not only natural, but rational. In this essay we explore the epistemology of that state of affairs. First we will canvass arguments for and against the claim that rational methods of reasoning must always reach the same conclusions from the same evidence. Then we will consider whether the acknowledgment that people have divergent rational reasoning methods should undermine one's confidence in one's own reasoning. Finally we will explore how agents who employ distinct yet equally rational methods of reasoning should respond to interactions with the products of each others' reasoning. We find that the epistemology of multiple reasoning methods has been misunderstood by a number of authors writing on epistemic permissiveness and peer disagreement.
\end{abstract}

[Forthcoming in Reasoning: Essays on Theoretical and Practical Thinking, M. Balcerak-Jackson and B. Balcerak-Jackson (eds.), Oxford University Press.]

Different people reason differently, which means that sometimes they reach different conclusions from the same evidence. We maintain that this is not only natural, but rational. In this essay we explore the epistemology of that state of affairs. First we will canvass arguments for and against the claim that rational methods of reasoning must always reach the same conclusions from the same evidence. Then we will consider whether the acknowledgment that people have divergent rational reasoning methods should undermine one's confidence in one's own reasoning. Finally we will explore how agents who employ distinct yet equally rational methods of reasoning should respond to interactions with the products of each others' reasoning. We find that the epistemology of multiple reasoning methods has been misunderstood by a number of authors writing on epistemic permissiveness and peer disagreement.

\section{Denying Uniqueness}

We claim that there are multiple, extensionally non-equivalent, perfectly rational methods of reasoning. Nowadays the opponents of this view rally behind what has come to be called "the Uniqueness Thesis". To understand our disagreement with them, and the arguments they make for their side, it will help to analyze exactly what the Uniqueness Thesis says.

When Richard Feldman introduced the Uniqueness Thesis in his (2007), he defined it as follows:

This is the idea that a body of evidence justifies at most one proposition out of a competing set of propositions (e.g., one theory out

Key words and phrases. rationality, reasoning, evidence, Uniqueness, permissivism, disagreement, epistemic standards. 
of a bunch of exclusive alternatives) and that it justifies at most one attitude toward any particular proposition. (p. 205)

Describing himself as "following Feldman," Roger White $(2005)^{1}$ argued for the Uniqueness Thesis, but defined it this way:

Given one's total evidence, there is a unique rational doxastic attitude that one can take to any proposition. (p. 445)

Those two theses do not say the same thing. In fact, Feldman's thesis says two distinct things (it's a conjunction), and White's thesis says something that is identical to neither of Feldman's conjuncts. The first thing Feldman says relates evidence to propositions, talking about which propositions are justified by a body of evidence. The second thing Feldman says relates evidence to attitudes. White's thesis then relates evidence to rational attitudes taken by people. So we really have three theses here:

Propositional Uniqueness: Given any body of evidence and proposition, the evidence all-things-considered justifies either the proposition, its negation, or neither.

Attitudinal Uniqueness: Given any body of evidence and proposition, the evidence all-things considered justifies at most one of the following attitudes toward the proposition: belief, disbelief, or suspension.

Personal Uniqueness: Given any body of evidence and proposition, there is at most one doxastic attitude that any agent with that total evidence is rationally permitted to take toward the proposition.

Propositional Uniqueness is not identical to the first conjunct of Feldman's Uniqueness Thesis, but is entailed by that conjunct. Attitudinal Uniqueness is Feldman's second conjunct. Personal Uniqueness is White's Uniqueness Thesis. ${ }^{2}$ We have framed the three theses in qualitative terms, assuming that the attitudes under study are belief, disbelief, and suspension of judgment. Analogous theses exist for other types of doxastic attitudes, such as quantitative degrees of belief. (Propositional: Given any body of evidence and proposition, the evidence confirms the proposition to a specific degree... Attitudinal: The evidence justifies at most one specific degree of belief in the proposition... etc.) In what follows we'll jump between these different framings of the theses depending on whether we're discussing full beliefs or credences.

The three theses are arranged in the order in which many epistemologists argue from one to another. Feldman, for instance, seems to think that a body of evidence justifies belief in a proposition only if it justifies that proposition (and justifies disbelief only if it justifies the negation, etc.). So he moves from Propositional

\footnotetext{
${ }^{1}$ Though Feldman's article was officially published in 2007, a draft had been circulating for a number of years before that. This explains how White could be "following Feldman" despite the fact that White's publication date came first.

${ }^{2}$ For reasons to prefer "at most one" formulations of Uniqueness, see (Kopec and Titelbaum 2016, pp. 190-1). Notice also that even if some attitude towards a proposition is rationally permissible for an agent, it might also be rationally permissible for that agent to adopt no doxastic attitude toward that proposition, for instance because she has never entertained it. Personal Uniqueness concerns only how many attitudes are permissible for an agent to adopt toward a proposition once she assigns it some attitude. To streamline argumentation we will set aside this complication and assume that all agents under discussion have assigned attitudes to all relevant propositions.
} 
Uniqueness to Attitudinal Uniqueness. Feldman then assumes that rationality requires an agent to adopt the attitude supported by that agent's total evidence, which takes him to something like Personal Uniqueness (though he doesn't include this conclusion as a conjunct of his official Uniqueness Thesis). In general, each thesis does seem necessary for the ones that come after. It's difficult to maintain that a unique attitude is rationally required of any agent with a particular body of evidence (Personal Uniqueness) without tracing that requirement back to a unique relation between the evidence and that attitude (Attitudinal Uniqueness). It is then difficult to establish a unique relation between the evidence and attitude without relying on some unique relationship between the evidence and the proposition toward which that attitude is taken (Propositional Uniqueness).

When first exposed to the Uniqueness debate, many philosophers intuitively reject the thesis on the grounds that it's too cognitively demanding - especially in its degree-valued formulations. Perhaps Attitudinal Uniqueness is true and for any body of evidence there is a unique credence that evidence supports. But can we really expect agents to perfectly discern that credence, down to arbitrarily many decimal points? While the relation of evidence to attitudes may be precise, agents should be granted a bit of leeway in approximating rational attitudes. If evidence $E$ justifies a credence in $H$ of exactly 0.7 , an agent could be rational while assigning $H$ a credence anywhere roughly in that vicinity.

This position denies Personal Uniqueness while leaving Attitudinal and Propositional intact. (It therefore shows that while Attitudinal Uniqueness may be necessary for Personal, it is not sufficient.) Similarly, one could outline a position that denies Personal and Attitudinal Uniqueness while leaving Propositional intact. But we wish to deny Uniqueness on a much deeper level - we deny Propositional Uniqueness (in both its qualitative and quantitative forms), and thereby deny all the forms of Uniqueness above. We do this because we don't believe there are evidential support facts of the sort Propositional Uniqueness implies.

How can one deny the existence of facts about evidential support? It's important to see exactly what sort of facts we're denying. Propositional Uniqueness asserts the existence of a two-place function defined over all pairs of propositions. Assuming any body of evidence can be represented as a conjunctive proposition, Propositional Uniqueness asserts the existence of a function that takes any ordered pair of evidence proposition and hypothesis proposition and returns what we might call a "justificatory status". ${ }^{3}$ (In the qualitative formulation that status is either justification of the proposition, anti-justification, or neither. In the degreed formulation the status is a numerical degree of support.) We're happy to admit that there may be some pairs of evidence and hypothesis that determine a justificatory status all on their own. At least one of the authors thinks this occurs in deductive cases: if the evidence entails the hypothesis, then it all-things-considered justifies that proposition; and if the evidence refutes the hypothesis then it justifies its negation. But deductive cases are a very special case among arbitrarily selected pairs of propositions. For many other evidence/hypothesis pairs, support facts obtain only

\footnotetext{
${ }^{3}$ If evidence is factive, then the conjunction representing an agent's total evidence must always be logically consistent, so the function under discussion need not be defined for ordered pairs containing inconsistent evidence propositions.
} 
relative to a third relatum; absent the specification of that third relatum, there simply is no matter of fact about whether the evidence justifies the hypothesis. ${ }^{4}$

The third relatum in question is a method of reasoning. Methods of reasoning are ways of analyzing evidence to draw conclusions about hypotheses. (We will also sometimes refer to them using White's (2005) and Schoenfield's (2014) terminology of "epistemic standards." ${ }^{5}$ ) Some methods of reasoning, while distinct, are extensionally equivalent: Given the same evidential inputs they will always yield the same outputs. For instance, you and I might both be perfect at addition, yet apply different algorithms in our heads to calculate sums. Yet many methods of reasoning are extensionally nonequivalent. We claim that a body of evidence supports a particular hypothesis only relative to a rational reasoning method that concludes that hypothesis from that evidence. And since there are multiple, extensionally nonequivalent rational reasoning methods, there isn't always a univocal fact of the matter about whether some evidence supports a particular hypothesis.

A version of this view is familiar to formal epistemologists: Subjective Bayesianism denies Propositional Uniqueness in exactly the manner we have been describing. In general, Bayesians hold that any rational agent can be represented as adhering to a particular "hypothetical prior" function $\mathrm{cr}_{h}$. The agent's credences at a given time can be obtained by conditionalizing her hypothetical prior on her total evidence at that time. A body of total evidence $E$ supports a hypothesis $H$ for the agent just in case $\operatorname{cr}_{h}(H \mid E)>\mathrm{cr}_{h}(H)$. Notice that facts about evidential support are therefore relative to the hypothetical prior of the agent in question. We can think of an agent's hypothetical prior as representing her epistemic standards - antecedent to the influence of any contingent evidence, the hypothetical prior encodes how an agent would respond to any package of evidence she might encounter, and which bodies of evidence she would take to support which hypotheses.

Some Bayesians - we'll call them "Objective Bayesians" - believe there is a unique rational hypothetical prior. ${ }^{6}$ In that case, whether a body of evidence supports a hypothesis is simply a matter of what that hypothetical prior says about the pair. So while evidential support is relative to that hypothetical prior, we need not treat it as an additional input to the evidential support function, since it will always have a constant value (so to speak). If there is only one rational hypothetical prior, Propositional Uniqueness is true. Yet many Bayesians ("Subjective Bayesians") believe multiple hypothetical priors are rationally acceptable. Two rational individuals could apply different hypothetical priors - representing extensionally nonequivalent epistemic standards - so that the same body of evidence supports a hypothesis for one of them but countersupports it for the other. For many proposition pairs, there simply are no two-place justification facts of the sort Propositional Uniqueness asserts.

Why would one take the seemingly-radical step of denying Propositional Uniqueness and admitting multiple perfectly rational, extensionally nonequivalent reasoning processes? Each author of this essay has his own reasons. Kopec (ms), roughly

\footnotetext{
${ }^{4}$ Compare the discussion at (Kelly 2014, pp. 308ff).

${ }^{5}$ Which may in turn be related to Lewis's (1971) "inductive methods".

${ }^{6}$ The "Subjective/Objective Bayesian" terminology has been used in a variety of ways in the Bayesian literature, and we don't want to wade into that history here. For purposes of this essay one can treat our use of these terms as stipulative. A classic example of an Objective Bayesian position in our sense is Carnap's early theory of confirmation in his (1950). Meacham (2014) uses the term "Impermissive Bayesianism" for what we are calling "Objective Bayesianism".
} 
speaking, views epistemic rationality as a subspecies of goal-oriented practical rationality. Among an agent's practical goals are various epistemic goals; it's then epistemically rational for the agent to hold those attitudes that constitute the most effective means of pursuing her epistemic goals. Different agents are permitted to have different epistemic goals, so rational agents may vary in the conclusions they draw from identical bodies of evidence.

Titelbaum (2010) argues that if there is a unique evidential support relation that extends beyond deductive cases, it must treat some predicates differently from others (think of "green" and "grue"). For agents to determine which bodies of evidence support which hypotheses, they must be able to differentiate the preferred predicates. If predicate preference must be determined from empirical facts, it will be impossible for agents to make that determination, since they must know which predicates are preferred before they can determine what empirical evidence supports. So one is left with either an extreme externalism on which agents cannot determine what their evidence supports, or an extreme apriorism on which preferred predicates, natural properties, or some such can be discerned a priori. Titelbaum would rather deny Propositional Uniqueness than adopt either of those other extreme positions. ${ }^{7}$

\section{Against Permissivism}

Roger White calls any position that denies the Uniqueness Thesis "permissivist". We will now review some of the arguments against permissivism. ${ }^{8}$

2.1. Consensus. Many anti-permissivist arguments are motivated by concerns about rational consensus. Feldman, White, and others have been very concerned with cases of interpersonal disagreement - cases in which agents disagree with their peers about some important matter despite possessing the same (relevant, total) evidence with respect to it. Feldman writes about two detectives on the same criminal case, White about members of a jury. There seems to be a deep concern that if permissivism is correct some such confrontations may be ultimately unresolvable.

Academics - like other professional seekers of information and understandingspend a great deal of time disagreeing with each other, citing evidence in an attempt to bring others along to their own point of view. If permissivism is true, there may be cases in which each of two disagreeing agents will say that she's responding to the available evidence in a perfectly acceptable manner, and each agent will be correct. This raises the specter of in-principle unresolvable disagreements, and may make us wonder why we put so much effort into convincing our peers.

This concern is related to a long-standing worry about Subjective Bayesianism. Philosophers of science have worried that if Subjective Bayesianism is correctif rational scientific inquirers may reason differently from the same experimental results - we will be hard-pressed to account for consensus among working scientists

\footnotetext{
${ }^{7}$ (Titelbaum 2010) argues against the existence of a three-place evidential support relation "evidence $E$ favors hypothesis $H_{1}$ over hypothesis $H_{2}$ ". This allows the argument to address contrastivist views which deny the existence of two-place evidential support relations ( $E$ justifies $H$ ) but accept such three-place relations. Since contrastivism will not be at issue in this essay, we will focus on arguments for and against a two-place relation.

${ }^{8}$ For a more comprehensive survey of arguments and motivations that have driven epistemologists to Uniqueness, see (Titelbaum and Kopec ms).
} 
about which experimental results support which hypotheses. Moreover, when disagreements arise as to the proper interpretation of results, no resolution may be available, as each party's reasoning may be perfectly rational. Subjective Bayesianism (and permissivism in general) seems to undermine a desirable objectivity in science. $^{9}$

When authors worry about consensus in science (and in reasoning more generally), it's often unclear which of a number of issues they are worrying about. First, they may be concerned to explain either descriptive or normative facts. Under the former heading, one wonders how to explain existing consensus in science about which theories are best supported by extant evidence. Unless scientists are byand-large competently tracking an evidential support relation constant for all of them, there seems no way to explain the large amount of scientific agreement we observe. ${ }^{10}$ Notice that we can draw a further distinction here about precisely what data is to be explained. Are we meant to explain the fact that different groups of scientists, after inter-group consultation, come to agree on which hypotheses are supported? Or must we explain the fact that different groups of scientists, without consulting, independently favor the same hypotheses on the basis of similar bodies of evidence? Call these phenomena "descriptive agreement after consultation" and "descriptive agreement in isolation".

An immediate response to these descriptive concerns is to deny that consensus is all that common among working scientists (thereby denying the putative phenomena to be explained). If there is objectivity to science, it is revealed not by actual scientists' opinions, but instead by our presumption that they would reach consensus under ideal conditions. ${ }^{11}$ This is a normative consensus concern - the notion that inquirers should draw the same conclusions from the same bodies of evidence. For instance, the great Subjective Bayesian L.J. Savage ${ }^{12}$ writes of his opponents,

It is often argued by holders of necessary and objectivistic views alike that that ill-defined activity known as science or scientific method consists largely, if not exclusively, in finding out what is probably true, by criteria on which all reasonable men agree. The theory of probability relevant to science, they therefore argue, ought to be a codification of universally acceptable criteria. Holders of necessary views say that, just as there is no room for dispute as to whether one proposition is logically implied by others, there can be no dispute as to the extent to which one proposition is partially implied by others that are thought of as evidence bearing on it. $(1954$, p. 67)

\footnotetext{
${ }^{9}$ Kelly (2008) characterizes this notion of objectivity explicitly in terms of agreement: "Objective inquiry is evidence-driven inquiry, which makes for intersubjective agreement among inquirers."

${ }^{10}$ For example, here's Earman (1992) complaining about Bayesian convergence results: "What happens in the long or the short run when additional pieces of evidence are added is irrelevant to the explanation of shared judgments about the evidential value of present evidence." (p. 149)

${ }^{11}$ cf. (Williams 1986, Ch. 8) and (Wright 1992).

${ }^{12}$ In the preface to his (1972), Bruno de Finetti wrote of the recently-deceased Savage that "it was clear how much was yet to be expected from his clarifying spirit for the success in our task: to relieve science and mankind from the strange superstitious prejudice that the obvious subjective probability feelings could or should be related to, or even replaced by, some hypothetical notion that, in some indefinable sense, could be called objective." (p. vi)
} 
Again, we can distinguish a norm that inquirers should agree in isolation from a norm that they should agree after mutual consultation. Later in this essay we will demonstrate how consensus after consultation (both descriptive and normative) can be achieved on a permissivist position. This will show that consensus after consultation concerns provide no compelling argument for Uniqueness.

That leaves the concern for normative consensus in isolation. But it's highly controversial that scientists in isolation working on the same evidence are rationally required to draw the same conclusion - the thesis would be severely contested by most historians and philosophers of science working in the wake of (Kuhn 1970). More to the point dialectically, the claim that reasoners working individually would, if rational, draw the same conclusions from the same evidence is tantamount to Personal Uniqueness. So it can hardly be used as a premise to argue for Uniqueness.

2.2. Justificatory arbitrariness. When confronted by the suggestion that rational agents with the same evidence might disagree because they have different epistemic standards (what he calls "starting points"), Richard Feldman writes,

Once people have engaged in a full discussion of issues, their different starting points will be apparent. And then those claims will themselves be open for discussion and evaluation. These different starting points help to support the existence of reasonable disagreements only if each side can reasonably maintain its starting point after they have been brought out into the open.... Once you see that there are these alternative starting points, you need a reason to prefer one over the other. (2007, p. 206)

It's interesting that Feldman poses this as a challenge about agreement after consultation ("Once people have engaged in a full discussion..."). Presumably, though, a more general point is being dramatized by the dialectical staging. If Uniqueness is true, exactly one method of reasoning is rationally correct, so there is no choice among methods for a rational agent to make. But if multiple methods are permissible it seems an agent must maintain the standard she does for some reason - the kind of reason she could cite in a confrontation with individuals employing different methods. The agent seems to need a reason not only to apply her own methods, but to prefer them to the other rational options. ${ }^{13}$

A permissivist may reply by denying that such reasons are required. On this line, an agent's epistemic standards constitute the point of view from which she evaluates reasons and evidence. That point of view cannot have - and does not need - evidential support. ${ }^{14}$

Alternatively, the permissivist may grant that reasons are required for applying one (rationally permissible) epistemic standard rather than another, but permit such reasons to be non-evidential. ${ }^{15}$ This approach nicely fits views on which an agent's methods of reasoning may depend on epistemic or practical goals. To get to Uniqueness, one needs not only the position that conflicting epistemic standards must be adjudicated on the basis of reasons, but also that such reasons must be

\footnotetext{
${ }^{13}$ By using words like "choice" and "maintain" we don't mean to suggest anything voluntaristic - an agent need not have chosen to adopt or maintain her epistemic standards at any particular point. An agent may possess a particular attribute (such as a moral code) for which she has reasons and for which she is justified despite never having explicitly chosen to adopt it.

${ }^{14}$ Compare (Schoenfield 2014, $\S 2.2$ ).

${ }^{15}$ cf. (Podgorksi 2016, p. 1928).
} 
evidential. After all, Uniqueness maintains that rational conclusions supervene on evidence; under Personal Uniqueness, rational agents with the same evidence will always draw the same conclusions. Thus Personal Uniqueness embodies a particularly strong form of evidentialism. ${ }^{16}$ A theorist already committed to such evidentialism will have reason to endorse Uniqueness, but again we've found a premise that is too close to the conclusion to provide an independent argument. And absent a commitment to strong evidentialism, it's difficult to see why an agent can't justify her choice of epistemic standards on non-evidential grounds. ${ }^{17}$

2.3. Causal arbitrariness. Feldman's concern above was a concern for justificatory arbitrariness - a concern that once an agent recognizes her method of reasoning is just one among the rationally-permitted many, she will be unable to maintain it without a specific kind of reason. Strictly speaking this is an attack on acknowledged permissivise cases, not permissive cases in general. An acknowledged permissive case is one in which not only are multiple rational methods available, but the agent also recognizes that fact. Epistemologists such as Stewart Cohen (2013) and Nathaniel Sharadin (2015) have suggested that while unacknowledged permissive cases are possible, acknowledged permissive cases are not. ${ }^{18}$ While it may be true that multiple methods of reasoning are rational in a particular case, recognizing that multiplicity may be corrosive to our epistemic practices.

We've just seen that if the corrosion is supposed to come from justificatory arbitrariness - the lack of reasons for maintaining one standard rather than anotherthe permissivist has responses available. But another kind of arbitrariness may be of concern: We may worry that permissivism allows epistemically arbitrary causal factors to influence a rational agent's beliefs. Katia Vavova nicely articulates the concern about arbitrary causal influences on belief:

The fact that you were raised in this community rather than that one is neither here nor there when it comes to what you ought to believe about God, morality, or presidential candidates. Yet factors like upbringing inevitably guide our convictions on these and other, less charged, topics. The effect is not always straightforwardperhaps you wouldn't be so liberal if you hadn't been raised in a liberal household, or perhaps you wouldn't be such a staunch atheist if your parents hadn't been so profoundly religious - but it is disturbing either way.... It's tempting to think that we should believe what we do because of evidence and arguments - not because of where we were born, how we were raised, or what school

\footnotetext{
${ }^{16}$ See (Kelly 2008), (Ballantyne and Coffman 2012), (Ballantyne and Coffman 2011), and (Kopec and Titelbaum 2016) for precise discussion of the logical relations between Uniqueness and various forms of evidentialism.

${ }^{17} \mathrm{~A}$ Uniqueness defender may allow the unique rational method of reasoning to be justified on the basis of both evidence and a priori considerations (with the assumption that such considerations do not vary across agents). Whether the a priori is itself evidential is a sticky issue. But either way, we wind up with the supervenience of rational conclusions on evidence and a strong evidentialist position.

${ }^{18}$ Though see (Ballantyne and Coffman 2012) for an argument that this position is unsustainable.
} 
we happened to attend. If that is right, however, and if such influences really are pervasive, then we are irrational in much of what we believe. $(\mathrm{ta})^{19}$

If Uniqueness is true, then all rational agents have the same (or at least extensionally equivalent) epistemic standards, so it doesn't much matter how they got them. But if conflicting epistemic standards are rationally permissible, which standards are possessed by a given rational agent will almost certainly be influenced by epistemically arbitrary causal factors. Once the rational agent recognizes this influence, it seems to undermine the rationality of the beliefs recommended by those standards.

The trouble with this as an objection to permissivism is that even if Uniqueness is true, epistemically arbitrary causal factors still influence a rational agent's beliefs. According to Uniqueness a rational agent's beliefs supervene on her evidence. But arbitrary factors (such as the ones Vavova lists above) can influence what body of evidence an agent possesses. Uniqueness defenders don't see this as a challenge to the view that rationality requires beliefs to be responsive to evidence. ${ }^{20}$ White, for instance, is highly sanguine about the chance events by which we come to have particular packages of evidence:

If I hadn't studied philosophy I would not believe that Hume was born in 1711. I would, if not disbelieve it, give little credence to that particular year being his birth date. And in fact I just learnt this fact by randomly flipping open one of many books on my shelf and reading where my finger landed. I was lucky indeed to be right on this matter! Of course there is nothing unsettling about this. There is nothing problematic about being lucky in obtaining evidence for one's belief. (2010, p. 597, emphasis in original)

And yet White is very concerned about the arbitrary events by which rational agents would come to have one epistemic standard rather than another if permissivism were true. Why the asymmetry?

We can develop a proposal for how White sees the asymmetry by noting a point he makes repeatedly in a number of his writings. Here it's important to understand that White believes in following one's evidence for a very different reason than Feldman does. In their co-authored work on evidentialism, Conee and Feldman write of their evidentialist thesis EJ, "We do not offer EJ as an analysis. Rather it serves to indicate the kind of notion of justification that we take to be characteristically epistemic - a notion that makes justification turn entirely on evidence... We believe that EJ identifies the basic concept of epistemic justification." (2004, pp. 83-84) At least when it comes to epistemic justification, Feldman takes the link between justification and evidence to hold on something like a conceptual level.

White, on the other hand, holds evidence significant for rationality and justification because of a particular feature evidence possesses: truth-conduciveness. White writes, "In inquiry my first concern is to arrive at a true conclusion regarding the defendant's guilt. And it is not clear why I should be so concerned with

\footnotetext{
${ }^{19}$ (White 2010), (Elga ms), (Schoenfield 2014), and (Schechter ms) also discuss the significance of agents' epistemic standards' being causally influenced by epistemically arbitrary factors.

${ }^{20}$ Ballantyne (2015) is very concerned about arbitrariness in the packages of evidence we receive, but he is not concerned to argue for Uniqueness.
} 
having my beliefs appropriately based unless this is conducive to the goal of getting things right." (2014, p. 316, emphasis in original) To remain neutral among various positions about what's epistemically important, we have been using the term "epistemically arbitrary" without precisely defining it. ${ }^{21}$ But it's fairly clear that for White causal processes are objectionably "arbitrary" when they have no tendency to pick out from among the standards available those that are more truthconducive. ${ }^{22}$ Now not every epistemologist agrees with White that rationality is so focused on truth. But the position is fairly common, and adopting it is not obviously identical to adopting the Uniqueness Thesis, so we will grant it arguendo to see where it leads. ${ }^{23}$

2.4. Evidence and truth. In that (2014) article White writes, "If there is evidence available strongly supporting one verdict, then it is highly probable that it supports the correct verdict" (p. 315); "In a non-permissive case where the evidence directs us to a particular conclusion, following the evidence is a reliable means of pursuing the truth" (ibid.); and "Common wisdom has it that examining the evidence and forming rational beliefs on the basis of this evidence is a good means, indeed the best means, to forming true beliefs and avoiding error." (p. 322) We could sum up these sentiments with the slogan "Most evidence isn't misleading." On the other hand, "In a permissive case... if either conclusion can be rationally held it would be natural to expect around a 50-50 split of opinions. In this case only about half of the inquirers will be correct in their conclusions." (p. 315) (This is why White repeatedly suggests that in a permissive case, applying a rationally permitted reasoning method would be no more likely to yield a true belief than flipping a fair coin.) So perhaps this is the key disanalogy: It's not distressing that an agent's particular batch of evidence was selected for her on the basis of arbitrary factors, because most batches of evidence rationally lead us to the truth. It is, however, distressing that if permissivism is true an agent's epistemic standards were selected for her on the basis of arbitrary factors, because she's got no better chance of reaching the truth by enacting those standards than if she had flipped a fair coin.

Epistemologists often say - both in print and in conversation - that most evidence isn't misleading. ${ }^{24}$ It is unclear to us not only why one should believe this slogan, but even what it is supposed to mean.

Start with the fact that for a permissivist, there will in many cases be no such thing as what a body of evidence supports on its own, so a fortiori there will be no facts about whether what the evidence supports is true. In a permissive case

\footnotetext{
${ }^{21}$ Notice that if "epistemically arbitrary" meant "arbitrary with respect to the evidence", the motivation for Uniqueness under consideration would be question-begging. The current question is whether it's rationally problematic for epistemically arbitrary factors to influence belief. If that were just the question whether it's rationally permissible for non-evidential factors to influence belief, answering it would simply be re-asserting one's position on Uniqueness.

${ }^{22}$ cf. (Vavova ta): "An irrelevant influence for me with respect to my belief $p$ is one that (a) has influenced my belief that $p$ and (b) does not bear on the truth of $p . "$

${ }^{23}$ For a different response to White's truth-conduciveness concerns about permissivism, see (Meacham 2014, §5.3).

${ }^{24}$ Just to select an example that happens to appear in the same volume as White's later Uniqueness piece (and with no intention to pick on this author in particular), Comesaña (2014, p. 240) baldly asserts, "If everything tells in favor of $H$ is true, then most likely $H$ is true" (where "everything" refers to an agent's total evidence).
} 
it's the pairing of a body of evidence and a method of reasoning that indicates conclusions, and it's that pairing that can be assessed for accuracy.

But let's see if we can support the slogan from a Uniqueness point of view, on which there are always facts about what conclusions a body of evidence supports on its own. The next question to ask is whether evidence is factive. If the point of asserting that most evidence isn't misleading is to advise an agent seeking truth to base her beliefs on rational conclusions from what she takes to be her evidence, then it's unclear whether we can assume all evidence is factive. After all, in evaluating that advice we might want to take into account that most of the agents applying it will be doing so on the basis of bodies of (what they take to be) evidence that include falsehoods.

Nevertheless, let's further grant the factivity of evidence so as to make the best case for the slogan we can. If evidence is factive, then at least evidence that entails a conclusion isn't misleading with respect to that conclusion. (Anything entailed by a truth is true!) Yet if White's goal in endorsing the slogan is to make evidence-following on a Uniqueness regime look more reliable than applying one's standards on a permissive view, entailing evidence isn't going to help him make that case. Any plausible permissivist view will require every rationally permissible epistemic standard to get the deductive cases right (at least if evidence is factive). For example, every hypothetical prior permitted by Subjective Bayesianism handles those cases correctly.

So now imagine Uniqueness is true, grant the factivity of evidence, and focus on non-deductive cases. What would we be asserting if we said that in most of those cases evidence is not misleading, and how might we support such a claim? First, the slogan involves a "most" claim, but suggests no particular measure over the infinite number of potential non-deductive evidential situations. Second, even once we've granted Uniqueness, any claim that evidence is non-misleading must still be relative - relative to the hypothesis we're wondering whether that evidence is misleading about. A given agent's body of total (factive) evidence is probably misleading with respect to some hypotheses and non-misleading with respect to others.

The slogan defender must therefore hold that for most non-entailing evidence/hypothesis pairs, the evidence supports the truth about that hypothesis. Presumably to avoid worries about counting the infinite space of such pairs, the sloganeer will back off to some claim about bodies of evidence actually possessed by real humans and hypotheses actually entertained by them. But even within this limited domain our evidence is often misleading in a systematic and widespread fashion. It's very plausible that, even when interpreted in perfectly rational fashion, humankind's total evidence concerning the physical behavior of the smallest bits of matter was hugely misleading for most of human history. (And it's probably the case that the bodies of evidence possessed by the majority of living humans are still misleading with respect to that domain.)

The best position for the defender of the slogan that most evidence isn't misleading is to maintain that with respect to everyday, useful hypotheses that come up in the ordinary course of life, most people possess bodies of evidence that generally aren't misleading. This fact helps explain why we tend to have true beliefs in that domain and are able to navigate the world as successfully as we do. 
The trouble is, the permissivist can give a similar defense of the claim that with respect to everyday, useful hypotheses that come up in the ordinary course of life, most people possess reasoning methods that (when applied to the bodies of total evidence they tend to have) generate beliefs that tend to be true. Not only is this claim explanatory in its own right; it may also be explainable by natural and cultural selection. These days whole areas of cognitive science tease out how humans are wired to process bodies of evidence they typically receive and explain why such coded heuristics might have helped us get things right in the environments in which we evolved. For instance, Bayesian vision scientists hypothesize that the human visual system employs "priors" that process retinal stimuli on the assumption that lighting sources come from above. ${ }^{25}$ This tends to be a fairly reliable assumption, and it's obvious why we might have evolved to make it.

In maintaining that typical reasoning methods are typically reliable,${ }^{26}$ the permissivist need not think that one unique method of reasoning is the most reliable (relative to typical bodies of evidence and typical hypotheses) and therefore rationally singled out. It's very plausible to maintain (especially given the counting difficulties involved) that a number of reasoning methods do roughly equally-well across typical evidence and hypothesis pairings, with some methods doing better on some occasions and some methods doing better on others. ${ }^{27}$

We began this discussion because White wanted to treat arbitrary-standards and arbitrary-evidence cases asymmetrically. Arbitrary evidence was not worrisome because most evidence points toward the truth, so even if your evidence is selected arbitrarily you're likely to get accurate results. On the other hand, White suggested that if multiple standards are rationally permissible "only about half of the inquirers will be correct in their conclusions." Yet to the extent we can make sense of the claim that most evidence isn't misleading, it looks equally plausible to say that most standards aren't misleading. ${ }^{28}$

Failing to consider - and then fully understand - the possibility that most permissible standards are truth-conducive is, perhaps, the most significant error made by participants on both sides of the Uniqueness debate. From the supposition that at least one rational reasoning method yields belief in a particular hypothesis and at least one yields belief in its negation (on the basis of the same body of evidence), many authors conclude that arbitrarily adopting a rational method gives the agent

\footnotetext{
${ }^{25}$ For citations see (Adams, Graf, and Ernst 2004). (Thanks to Farid Masrour for help with this reference.)

${ }^{26}$ In fact, the permissivist need only maintain that typical rationally-permissible reasoning methods are typically reliable.

${ }^{27}$ We mention here only to reject as irrelevant the hypothetical prior that is guaranteed to have the highest reliability possible. Consider a hypothetical prior that, relative to any factive body of evidence, assigns credence 1 to every proposition that's true in the actual world, and credence 0 to every proposition that's actually false. While such a prior could certainly be defined - and God could even write out its values - it doesn't represent a method of reasoning available in any meaningful sense to non-omniscient folk. Thus its existence doesn't call into question the rational permissibility of reasoning methods we might actually employ that are admittedly less accurate.

${ }^{28}$ We've been treating the slogan that most evidence isn't misleading as asserting a contingent, empirical truth. Yet there are views of evidence/rationality/justification on which the slogan can be defended a priori. These include reliabilist theories of justification, and some semantic responses to skepticism (e.g. (Putnam 1981) and (Chalmers 2007, esp. §7)). Suffice it to say that if these approaches provide arguments for the slogan that most evidence isn't misleading, they will also provide arguments for the position that most rationally-permissible methods of reasoning aren't misleading either.
} 
a fifty-fifty chance of believing the truth. ${ }^{29}$ This is like learning that a bin contains at least one red and at least one green jellybean, then concluding that randomly selecting a bean must yield an equal chance of each color. Whether that's true depends not only on the randomness of the selection process, but also on the overall contents of the bin. If epistemically arbitrary causal factors select standards for you from a set most of whose members are reliable, the fact that your standards were arbitrarily selected from that set is no reason to question their reliability.

Here's where we stand dialectically: Either the thesis that most evidence isn't misleading can be established (on some plausible interpretation), or it cannot. If it cannot, then epistemically arbitrary causal influences are a rampant problem for all truth-centric approaches to rationality, whether they ascribe to Uniqueness or not. If the thesis can be established, then we ought to be able to establish on similar grounds that most rational reasoning methods aren't misleading. ${ }^{30}$ Once more, epistemically arbitrary causal influences on reasoning methods will provide no more of a problem than arbitrary influences on evidence. Either way, causal arbitrariness provides no special problem for permissivists. ${ }^{31}$

But we think the epistemological significance of multiple rational truth-conducive methods of reasoning is even greater than that.

\section{The Reasoning Room}

In the previous section we offered some (admittedly fairly armchair) reasons to believe that in most everyday situations, most of the methods of reasoning employed by rational people will be generally truth-conducive, even if some of those methods are extensionally non-equivalent. But even if that's not true in most everyday situations, it is certainly true in some situations. For instance, though the scientific groups working with the IPCC (International Panel on Climate Change) use different methods of analysis, and often arrive at different predictions for the future of the climate, to the extent we can discern these things (using cross-validation

\footnotetext{
${ }^{29}$ Like White, Schechter (ms, p. 7) assumes that if an agent's epistemic standards were selected in an arbitrary fashion, that agent is unlikely to have reliable standards. A similar assumption is made by Premise P2a of (Ballantyne 2012), which deems a belief irrational if there's even one nearby possible world in which the agent reached the opposite conclusion based on the same evidence and cognitive capacities. Schoenfield (2014) — who argues for permissivism! - concedes to White that permissivism will undermine the truth-conduciveness of epistemic standards in the mind of anyone who doesn't already subscribe to one of those standards. And in a similar vein, Dogramaci and Horowitz (2016) write, "under permissivism... rational reasoners cannot be ensured to be as reliable as they can be if uniqueness is true."

${ }^{30}$ One might wonder who's supposed to be doing all this establishing-must the agent know that most reasoning methods are reliable in order for it to be rational for her to apply one? (cf. White's discussion of "sticky pills" at (2005, p. 449)) This question comes up for the reliability of evidence as much as it comes up for the reliability of methods of reasoning. But more importantly, this is a standard question in epistemology — need one know a method is reliable before it can yield justified beliefs? - to which there are now many standard replies. Perhaps it's enough for the methods just to be predominantly reliable, even if the agents who employ them cannot establish that fact. Or perhaps agents possess default warrant to believe their methods are reliable absent any concrete evidence to the contrary (compare (Wright 2004)). Notice that if we follow this line, learning that one's methods were selected by an epistemically arbitrary causal factor need not supply a defeater for the claim that one's methods are reliable; a true defeater would also have to establish that the set selected from was not predominantly reliable.

${ }^{31}$ (Schoenfield 2014) offers an alternate response to the causal arbitrariness attack on permissivism. Since she has granted White's assumption that arbitrarily-selected standards must be unreliable, her response is to reorient the debate away from an exclusive focus on truth.
} 
and the like) it seems that each of them is generally reliable. So there's practical significance in asking questions about the epistemology of such groups, such as: Should an agent's awareness that her reasoning methods are just one of a number of rationally-permitted, equally-reliable methods undermine the conclusions of her reasoning? Should such an agent alter her opinions if she encounters another rational agent who's drawn opposite conclusions?

A number of epistemologists have made strong claims about how these questions should be answered in all permissive situations. We want to show that those answers misdiagnose permissive situations containing divergent but widely reliable reasoning methods. To make our case, we will focus on a highly artificial, highly regimented reasoning situation. Like many philosophical examples, this situation allows us to make efficient progress by reducing the number of unknowns and messy moving parts. Nevertheless, we believe the core epistemic features of the situation are shared with many real-life examples, and so allow us to draw important lessons for reasoning in real life. ${ }^{32}$

Here's the situation:

You are standing in a room with nine other people. Over time the group will be given a sequence of hypotheses to evaluate. Each person in the room currently possesses the same total evidence relevant to those hypotheses. But each person has a different method of reasoning about that evidence.

When you are given a hypothesis, you will apply your methods to reason about it in light of your evidence, and your reasoning will suggest either that the evidence supports belief in the hypothesis, or that the evidence supports belief in its negation. Each other person in the room will also engage in reasoning that will yield exactly one of these two results.

This group has a well-established track record, and its judgments always fall in a very particular pattern: For each hypothesis, 9 people reach the same conclusion about which belief the evidence supports, while the remaining person concludes the opposite. Moreover, the majority opinion is always accurate, in the sense that whatever belief the majority takes to be supported always turns out to be true.

Despite this precise coordination, it's unpredictable who will be the odd person out for any given hypothesis. The identity of the outlier jumps around the room, so that in the long run each agent is odd-person-out exactly $10 \%$ of the time. This means that each person in the room takes the evidence to support a belief that turns out to be true $90 \%$ of the time.

We submit that in the Reasoning Room, it is rationally permissible for you to form the belief your reasoning method suggests is supported by the evidence. The same goes for each other agent in the room. And since at least one of those agents disagrees with you about what belief the evidence supports, this means that at

\footnotetext{
${ }^{32}$ See, for instance, the case study in (Hicks 2015) of debates over yields of genetically modified crops. Hicks ultimately attributes the controversy to differing "epistemological standards" among the interlocutors, who "have radically different ideas... about what kinds of research should be carried out in order to support or undermine a claim." (p. 2)
} 
least one agent is rationally permitted to adopt a belief that disagrees with yours. So we are interpreting this example as a permissive case. ${ }^{33}$ (Later we'll discuss how Uniqueness defenders might re-interpret the example.) Interpreted that way, the Reasoning Room is a case in which you and another agent have extensionally nonequivalent, rationally permissible methods of reasoning about a particular kind of evidence, yet each of those methods is truth-conducive in the long run.

Interpreted permissively, the Reasoning Room puts the lie to a number of claims that have been made about the epistemology of permissivism. For instance, at one point White writes,

Supposing [permissivism] is so, is there any advantage, from the point of view of pursuing the truth, in carefully weighing the evidence to draw a conclusion, rather than just taking a belief-inducing pill? Surely I have no better chance of forming a true belief either way. If [permissivism] is correct carefully weighing the evidence in an impeccably rational manner will not determine what I end up believing; for by hypothesis, the evidence does not determine a unique rational conclusion. So whatever I do end up believing upon rational deliberation will depend, if not on blind chance, on some arbitrary factor having no bearing on the matter in question.

(2005, p. 448)

If you behave in Reasoning Room the way we have described, which belief you adopt may depend on an arbitrary factor with no bearing on the matter in question. Nine of you in the room will adopt one belief while the last adopts the opposite; all of you were assessing the same evidence; whatever caused you to have divergent methods of reasoning was not a function of the evidence. It's also true that in this example the evidence does not determine a unique rational conclusion (because at least two rational people in the room reached opposite conclusions from that evidence). Yet it doesn't follow that weighing the evidence in a rational manner has not determined what you ended up believing. After all, if you had made a reasoning mistake and misapplied your methods to that same evidence, you would've wound up believing something else. And it certainly does not follow that there is no advantage "from the point of view of pursuing the truth" to weighing the evidence over randomly taking a belief-inducing pill. Weighing the evidence according to your standards gives you a $90 \%$ chance of believing the truth, while taking a beliefinducing pill would give you only a $50 \%$ chance.

White's comparing reasoning in a permissive case to pill-popping is another way for him to suggest that any epistemically arbitrary choice among rival epistemic standards must leave the agent with a low probability of accurate belief. But we noted earlier that an arbitrary or chancy selection among a number of options, most of which are reliable, yields a high probability of believing truths. In the Reasoning Room, carefully weighing the evidence after arbitrarily selecting one of the available epistemic standards would leave you no better off with respect to the truth than

\footnotetext{
${ }^{33}$ It's important to note that our position here is stronger than what Podgorksi (2016) calls "dynamic permissivism". For Podgorski, permissivisn is true because different agents are permitted to consider (i.e., reason about) different proper subsets of their evidence. Since these distinct subsets may point in different directions, Podgorski thinks there can be cases in which rational agents with the same total evidence reason to contradictory conclusions. We read the Reasoning Room as permissive in a much stronger sense: we take it that the agents in the room may rationally draw conflicting conclusions from their total evidence.
} 
popping a pill that gave you a $90 \%$ chance of accurate belief. If truth-conduciveness is our sole consideration, that's not a very good objection to permissivism. ${ }^{34}$

White does consider the possibility that epistemic standards could be reliable without being rationally unique. He writes:

It might be suggested that rationally evaluating the evidence is a fairly reliable means of coming to the correct conclusion as to whether $P$, even if that evidence does not determine that a particular conclusion is rational. But it is very hard to see how it could.... ${ }^{35}$

Even if it is granted that a rational person needn't suspend judgment in such a situation, just how rational evaluation of the evidence could reliably lead us to the truth in such a case is entirely mysterious. It would have to be by virtue of some property of the evidence whose reliable link to the truth is inaccessible to the inquirer. For if an inquirer is aware that the evidence has feature $F$, which is reliably linked to the truth of $P$, then surely it would be unreasonable to believe $\sim P$. It is hard to imagine what such a truth-conducive feature could be, let alone how it could act on an inquirer's mind directing him to the truth. (ibid.)

We maintain that in the Reasoning Room, the evidence (alone) does not determine that a particular conclusion is rational. That's because we view the Reasoning Room as a permissive case, and in permissive cases evidence favors hypotheses only relative to particular methods of reasoning. Yet it is not mysterious in this case how rational evaluation of the evidence reliably leads the agents involved to the truth, and rational evaluation does not do so by virtue of some property whose reliable link to the truth is inaccessible to the inquirer.

Recall the IPCC groups. Each of them applies a particular analysis technique to available climate data, checking whether that data has particular features, then using those features to make a prediction. Or think about the ten individual agents in the room, applying their idiosyncratic methods of reasoning. Perhaps one of them evaluates the hypothesis $H$ by virtue of how it trades off simplicity with fit to the evidence. Or perhaps another agent leans toward $H$ on the basis of a particular statistical significance test. As she applies that test, the relevant features of the evidence and hypothesis are perfectly accessible, and it's not mysterious how such a test could reliably point her toward the truth (even if other tests might point her in a different direction).

The Reasoning Room also allows the permissivist to address the distinction between permissive cases and acknowledged permissive cases. In the example it is rationally permissible to adopt the belief that your reasoning suggests is supported by the evidence. At the same time, you are absolutely certain there is at least one person in the room whose reasoning pointed her in the opposite direction. Following her reasoning is just as rationally permissible for her as following your reasoning

\footnotetext{
${ }^{34}$ Of course, we could always up the number of agents in the Reasoning Room to bring the long-run reliability score as arbitrarily close to $100 \%$ as we'd like. Upping the numbers might also make some readers more comfortable with our conclusion that it's rationally permissible for you to adopt the belief your reasoning says the evidence supports.

${ }^{35}$ In one of the elided sentences White once more baldly asserts, "Evidence can be misleadingi.e. point us to the wrong conclusion-but this is not common."
} 
is for you. So not only do we have two agents in the room who have rationally drawn opposite conclusions from the same evidence; each of them is aware of the existence of a person (indeed, a very nearby person!) with rational beliefs different from her own. Nevertheless, it remains rationally permissible for each agent to maintain her own opinions. ${ }^{36}$ Contra Cohen and Sharadin, it's possible to have not only permissive cases but acknowledged permissive cases.

Now something different would happen if the two agents we were just discussing actually met and began to exchange views. Suppose your reasoning method suggests that your total evidence supports belief in $H$. So you form a belief in $H$. You then randomly select another occupant of the room, and ask her what she concluded. Suppose she tells you that as recommended by her reasoning method, she believes $\sim H$. We submit that it would then be rational to suspend judgment as to the truth of $H$.

Here's an intuitive explanation why: Given what you know about the distribution of opinions in the room, you should expect before interacting with your colleague that she will agree with you about the hypothesis. Before interacting you believe $H$, so you believe $8 / 9$ of the people in the room also believe $H$, so you expect a randomly selected peer to agree with you. When you find that she believes $\sim H$ instead, this is a surprising result, which leads you to take much more seriously the possibility that you are the only $H$-believer in the room. So it would be reasonable for you to suspend judgment on $H$.

For those who'd like a more precise argument, we offer a credal version of the Reasoning Room. Suppose the setup of the room is that for each hypothesis delivered, your reasoning will suggest either that the evidence supports a credence of 0.9 in the hypothesis or a credence of 0.1 . You then (rationally permissibly) adopt the credence your reasoning says the evidence supports. ${ }^{37}$ Suppose, for instance, that you assign credence 0.9 to $H$. You then randomly select another occupant of the room, and find that her reasoning led her to a 0.1 credence in $H$. At that point, some basic Bayesian reasoning will lead you to a credence of 0.5 in $H .^{38}$ This is the credal analog of suspending judgment. ${ }^{39}$

\footnotetext{
${ }^{36}$ cf. (Podgorksi 2016, p. 1931). Notice also that if we added to the Reasoning Room that the ten reasoning methods were somehow arbitrarily shuffled and assigned to the agents at random, we would have an explicit case in which being aware that your standards are arbitrarily assigned does not defeat the attitudes endorsed by those standards.

${ }^{37}$ The credal case allows us to say more about why it's a good idea to adopt the attitude your reasoning says the evidence supports. In Bayesian terms, this policy has the advantage of being perfectly "calibrated" in the long run. Moreover, if we measure accuracy by a proper scoring rule, it's the policy that maximizes long-run expected accuracy.

${ }^{38}$ Let $D$ be the proposition that a randomly-selected member of the room disagrees with your assignment. By Bayes' Theorem we have

$$
\begin{aligned}
\operatorname{cr}(H \mid D) & =\frac{\operatorname{cr}(D \mid H) \cdot \operatorname{cr}(H)}{\operatorname{cr}(D \mid H) \cdot \operatorname{cr}(H)+\operatorname{cr}(D \mid \sim H) \cdot \operatorname{cr}(\sim H)} \\
& =\frac{1 / 9 \cdot 9 / 10}{1 / 9 \cdot 9 / 10+1 \cdot 1 / 10}=\frac{1 / 10}{2 / 10}=\frac{1}{2}
\end{aligned}
$$

${ }^{39}$ What if your randomly-selected peer turns out to have the same credence as you in $H$ ? Learning of her credence should increase your confidence in the hypothesis above 0.9. (In fact, your credence should go all the way to 1!) This is an instance of an effect noted independently by Casey Hart and by Easwaran, Fenton-Glynn, Hitchcock, and Velasco (2016). (The latter call the effect "synergy".) It's also another way of showing that learning $D$ should decrease your
} 
The Reasoning Room therefore refutes Thomas Kelly's claim that if permissivism is true, there can be no reason for an agent to change her attitudes upon encountering a peer who disagrees. ${ }^{40}$ Kelly argues for this claim by describing a case in which I assign a credence of 0.7 to a hypothesis on the basis of my evidence, while admitting it would be equally reasonable to assign a slightly lower credence to that hypothesis on the basis of the same evidence. You, meanwhile, assign a credence slightly lower than 0.7 to the hypothesis on the basis of that evidence, while admitting it would be equally reasonable to assign exactly 0.7 . We then meet and exchange views.

Responding to the suggestion that after the exchange we should adjust our credences towards each other's, Kelly writes,

That seems wrong. After all, ex hypothesi, the opinion that I hold about [the hypothesis] is within the range of perfectly reasonable opinion, as is the opinion that you hold. Moreover, both of us have recognized this all along. Why then would we be rationally required to change? (2010, p. 119)

The Reasoning Room provides a straightforward answer to Kelly's rhetorical question. ${ }^{41}$ In the credal version of the example you initially assign one credence while being perfectly aware that at least one individual in the same room (entirely rationally) makes the diametrically opposite assignment. Upon randomly selecting an individual from the room and finding out that she made that opposite assignment, it's rational for you to split the difference between her initial credence and yours. This does not require denying that either her initial assignment or yours was rational given the evidence each of you had at that time. It merely requires admitting that in light of your new total evidence (which includes information about the attitudes of your randomly-selected peer), the probability of $H$ is $1 / 2$. This change is motivated not by finding any rational fault in one's previous attitude, but instead by coming to have evidence that makes a new attitude look more accurate, or truth-conducive. ${ }^{42}$

confidence in $H$. Since your current confidence in $H$ is a weighted average of the confidence you'd assign if you learned $D$ and the confidence you'd assign if you learned $\sim D$, the fact that the latter is higher than your current confidence means that the former must be lower.

${ }^{40}$ A similar suggestion seems to be made by Feldman at (2007, pp. 204-5). White (2010, n. 7) also writes, "If we really think there are [epistemically permissive] cases then even meeting an actual disagreeing peer seems to pose no challenge to one's belief." We will focus on Kelly because he goes on to provide an argument for his claim. (Thanks to (Ballantyne and Coffman 2012) for the additional citations.)

${ }^{41}$ As Christensen (2016) notes, there are two importantly different kinds of peer disagreement cases. The peer disagreement literature often proceeds under the assumption of Uniqueness, and so assumes that when individuals with the same evidence disagree it must be because one of them has made a mistake in applying the correct epistemic standards to that shared evidence. (This is why peer disagreement cases are often analyzed alongside cognitive malfunction cases.) But in permissive cases there can also be disagreement between agents who have applied their standards correctly to the same evidence, yet happen to have differing epistemic standards. This is the type of case Kelly considers, and the type of case we will be discussing. (For what it's worth, Titelbaum's (2015) argument against conciliating in peer disagreement cases applies only to the other type of disagreement, in which the disagreeing parties share epistemic standards.)

${ }^{42}$ Ballantyne and Coffman (2012) argue against Kelly that it can make sense to split the difference upon encountering a disagreeing peer in a permissive case if neither of the parties initially realized that the case was a permissive one. Christensen (2009) argues that splitting the difference may be sensible when an agent doubts she has applied her own epistemic standards 
The Reasoning Room also refutes a claim made by Stewart Cohen (among oth$\left.\mathrm{ers}^{43}\right)$. Cohen writes,

Note that I do not need to encounter a peer at a different credence for there to be accuracy pressure on my credence. Simply recognizing a rational credence different from my own is enough to undermine the rationality of my credence.... In such a case, the same pressure exists to revise in the direction of the other credence. (2013, p. 103, emphases added)

In the credal Reasoning Room you are certain before interacting that another rational agent assigns a different credence than your own. This exerts no pressure on you to change your credence of 0.9 . Yet actually encountering that rational peer pressures you to drop your credence to 0.5. There can be a significant difference between knowing one is in a permissive case and actually uncovering a particular individual with whom one disagrees. It's one thing to know that at least one person in a room disagrees with you. It's another thing to randomly select a peer and find that she disagrees. Such an encounter suggests that disagreement might be not just present, but representative, in which case your opinions should change.

We have just seen that if our interpretation of the Reasoning Room is correct, the example accomplishes a number of important things: it refutes a number of charges made against permissivism by White and others, it establishes the possibility of acknowledged permissive cases, and it shows that conciliating in the face of peer disagreement can be compatible with permissivism. Our interpretation assumes that the Reasoning Room is a permissive case, which runs counter to the Uniqueness Thesis. So how might a Uniqueness defender respond to the example? There are a couple of options. First, the Uniqueness theorist might agree that the ten agents in the Reasoning Room all apply different methods of reasoning to the same evidence. In that case (the Uniqueness theorist will say), at most one of those methods is the uniquely correct reasoning method, and when the agents differ in their attitudes toward $H$ at least one of them is irrational in doing so. While this response is available to the Uniqueness defender, it is not particularly interesting at this stage of the dialectic. The point of the Reasoning Room is to demonstrate that if one adopts a permissivist reading of the example, then various conclusions often imputed to permissivism need not follow. Simply denying permissivism as it applies to the example misses the point.

But there's a second, more interesting response available. The Uniqueness theorist might argue that the agents in the reasoning room reach different conclusions about $H$ not simply because they have different reasoning methods, but because

rationally. The Reasoning Room establishes the stronger thesis that splitting the difference can be rational even in antecedently-acknowledged permissive cases where both parties know no rational error has occurred.

Notice also that splitting the difference in the Reasoning Room doesn't involve rejecting one's old epistemic standards and somehow adopting new ones. Instead, you have a constant set of epistemic standards throughout the example that recommend one attitude towards $H$ before any interaction has occurred, then a different attitude if particular evidence about that interaction comes to light. The epistemic standards one applies in isolation, while yielding different results than someone else's standards in isolation, may nevertheless direct one to reach agreement with that someone after consultation.

${ }^{43}$ See, for instance, (Kelly 2005, §5). 
they are responding to different bodies of total evidence. When you are given hypothesis $H$ to consider, reason through your evidence, and judge that it supports belief in $H$, your total evidence comes to include the fact that you have reasoned from the original evidence to $H$. This fact is not possessed by the other agents in the room, so your total evidence differs from theirs. Most importantly, your total evidence differs from that of an agent who has reasoned from the original evidence to $\sim H$. (Meanwhile that agent possesses evidence you lack about the judgment rendered by her own reasoning. ${ }^{44}$ ) Unlike the first Uniqueness defender, this Uniqueness theorist grants that the varying attitudes adopted towards $H$ by the agents in the Reasoning Room are rationally permissible. But those differing attitudes are permissible because they are assigned relative to different bodies of total evidence. So the distinction between Uniqueness and permissivism plays no role in the Reasoning Room, and the example demonstrates nothing about the commitments of permissivism.

Again, we have to be careful about the dialectic here. The permissivist offers the Reasoning Room as a case in which the agents' differing reasoning methods lead them to different conclusions, while the Uniqueness theorist attributes the different conclusions to differences in total evidence. Depending on one's definition of "evidence", one could squabble about whether facts concerning one's own reasoning may count as evidence. But we prefer to avoid such definitional squabbles by noting that the really important question is whether facts about one's own reasoning are part of one's relevant total evidence. Out of all of an agent's evidence, only what's relevant to a hypothesis may rationally influence her attitudes, and that relation is determined by the agent's epistemic standards. To deny that the Reasoning Room illustrates permissivist commitments, the Uniqueness theorist must establish in a manner acceptable to permissivists that all rationally-permissible epistemic standards treat facts about one's own reasoning concerning a hypothesis $H$ as evidence relevant to $H{ }^{45}$ That strikes us as a tall order.

In fact, matters are even worse for the Uniqueness defender. Because it seems to us that if one is going to take a restrictive view of what's rationally permissible in the Reasoning Room, one ought to reach the conclusion that each agent's evidence about her own reasoning is not relevant to determining her attitude toward $H$. To see why, let's very carefully review who has what evidence at what times in the example. Initially, before the hypothesis is provided and any reasoning is performed, everyone in the room shares a common body of total evidence we'll call $E$. You then receive the hypothesis $H$, reason about it, and judge that $E$ supports belief in $H$. At that point your total evidence is $E^{\prime}$ : the conjunction of $E$ with the fact that

\footnotetext{
${ }^{44}$ Though he doesn't endorse it, Feldman discusses the proposal that an agent's "strong sense or intuition or 'insight' that the arguments, on balance, support her view" counts as evidence for that agent. (Feldman 2007, p. 207) He attributes a similar idea to (Rosen 2001, p. 88).

${ }^{45}$ Just to be crystal clear why this conclusion is required: Suppose there are at least two distinct rationally permissible epistemic standards that treat facts about $H$-reasoning as irrelevant to $H$. Then we could build a Reasoning Room case in which agents with those two standards reach different conclusions about $H$, and the differences would not be attributable to the differences in their total evidence generated by their awareness of their own reasoning. (Perhaps the Uniqueness theorist could triumph by arguing that even if there are many permissible standards, only one of them treats facts about $H$-reasoning as irrelevant to $H$. But that seems an awfully implausible position.)
} 
you have judged $E$ to support $H{ }^{46}$ In the meantime, at least one of your peers in the room has taken $E$, reasoned about it, and concluded that $E$ supports belief in $\sim H$. So her total evidence is $E^{*}$ : the conjunction of $E$ with the fact that she has judged $E$ to support $\sim H .{ }^{47}$

If Uniqueness is true, there must be a fact of the matter about whether $E$ supports belief in $H$ or $\sim H$. Let's suppose (without loss of generality) that in fact, $E$ supports belief in $\sim H$. In other words, your reasoning has led you to a false judgment about what $E$ supports. In order for the Uniqueness supporter to accept as rational the attitudes we've suggested for each agent at each stage of the example, the Uniqueness supporter will have to say that although $E$ supports belief in $\sim H$, your belief in $H$ after engaging in your reasoning is rational because $E^{\prime}$ supports belief in $H$. In other words, while $E$ points to belief in $\sim H$, your falsely judging the opposite, then adding a fact about the content of that judgment to your total evidence, makes it rational for you to believe $H$.

This is a truly bad idea. Our Uniqueness theorist has now embraced a curious theory of evidential bootstrapping, on which an agent, by falsely judging that her total evidence supports some conclusion, can thereby make it the case that her (new) evidence does indeed support that conclusion. While this is bad enough, consider further your attitude, after performing your reasoning, toward the proposition that $E$ supports belief in $H$. What attitude toward this second-order proposition is supported by $E^{\prime}$ ? If $E^{\prime}$ supports belief in this proposition, then we have a false proposition made rational to believe by the fact that you have judged it to be true. On the other hand, if $E^{\prime}$ does not support belief in the second-order proposition, ${ }^{48}$ then you continue to rationally believe $H$ on the basis of a judgment that your current evidence does not endorse. ${ }^{49}$

None of these positions is absolutely indefensible, but all of them seem tremendously awkward. Moreover, a Uniqueness defender need not accept them in order to maintain the Uniqueness Thesis. The Uniqueness defender bites these bullets only if she insists that were there any permissive cases, the Reasoning Room would

\footnotetext{
${ }^{46}$ It's significant here that as we envision the Reasoning Room scenario (in both its belief and credence versions), your initial determination about $H$ is made entirely on the basis of firstorder evidence $E$. The facts in the example about the track-records of the individuals involved (including yourself) are there only to help you recognize that you're in a permissive case, and to drive your reaction to the discovery of a disagreeing peer. If we wanted we could purify this issue by structuring the example so that you gain the track-record information only after forming a judgment about how your first-order evidence bears on $H$.

${ }^{47}$ One might worry that this reading assumes a great deal of introspection on your part: That whenever you judge a body of evidence to support a hypothesis, you at the same time notice that you have done so, and the fact that you have done so is added to your evidence. The Uniqueness theorist's reading of the example could be defanged by suggesting that this sort of introspective awareness isn't always present, and by stipulating that the Reasoning Room is one case in which it isn't. But it seems to us that the Uniqueness theorist's reading is already a bad idea independently of this consideration, so we won't further pursue the introspection line here.

${ }^{48}$ As (Titelbaum 2015) argues it cannot.

${ }^{49}$ Here's another reason why this reading is a bad idea: We usually think that if something is an important piece of evidence for a conclusion, that evidence can be explicitly cited in favor of the conclusion. In the case at hand a crucial piece of evidence for $H$ is the fact that you have judged $E$ to support $H$ (after all, without that fact in the body of total evidence, your evidence didn't support $H$ ). Yet would anyone ever cite, as part of their evidence for a hypothesis, the fact that they themselves judged their evidence to support it?
} 
not be one of them (so that the Reasoning Room cannot be used to assess the commitments of permissivism). Whether it's worth it for the Uniqueness theorist to make this move depends on what motivates her to believe in Uniqueness. For example, a Uniqueness defender driven by concerns about objectivity and/or consensus will not want the proposed reading of the Reasoning Room. Suppose we maintain Uniqueness for the Reasoning Room by counting facts about an agent's reasoning on a hypothesis as evidence relevant to that hypothesis. Then why not apply the same reading to rational scientific inquirers operating in isolation on the same body of empirical data? The moment one scientist has a thought about the significance of that data not shared by the other inquirers, her evidence will diverge from theirs and allow her to (rationally) reach different conclusions. The Uniqueness defender's motivating thought that rational scientists confronted with the same data should draw the same conclusions will fall by the wayside..$^{50}$

\section{Conclusion}

The forgoing discussion has revealed a great deal about the epistemology of divergent reasoning methods. While we cannot conclusively establish that real-life reasoning methods are generally reliable, we have seen that cases in which extensionally nonequivalent rational methods are reliable provide important counterexamples to many charges that have been made against permissivism. Such cases also show that arbitrary causal influences on methods of reasoning need not be undermining, and may help explain why rational inquirers come to agreement after consultation.

Might there be other reasons for an agent to worry about the possibility that reasoning methods distinct from her own might yield opposing rational conclusions? We will close by raising one more idea that we've sensed floating through the Uniqueness literature.

Concerns about objectivity often mask concerns for authority. Permissivism (especially in acknowledged permissive cases) requires the agent to maintain a sort of equanimity about the variety of rationally permissible methods of reasoning. Yet while recognizing that her own methods are but one rationally permissible option among many, the agent is nevertheless supposed to treat those methods as authoritative - normative for her own case. Permissivism seems to create a tension between respecting other methods as equally valid and ceding the necessary authority to one's own. ${ }^{51}$

It's important not to commit a level confusion here. Agents adopt doxastic attitudes towards propositions - propositions that often concern objective facts in the world, beyond any ability of the agent to affect their truth-value. But the attitude adopted (belief or disbelief, high or low credence) is a subjective feature of the agent, not part of the attitude's propositional content. It does not automatically follow from the objectivity of what's believed that there is any objectivity to the norms for belief.

\footnotetext{
${ }^{50}$ Since White seems very much motivated by consensus concerns, he should be uncomfortable with this Uniqueness-consistent reading of the Reasoning Room. White also endorses the principle that "a belief can always rationally survive learning the epistemic value of one's evidence." (2005, p. 450) Yet it does not seem under this Uniqueness reading of the Reasoning Room that your belief in $H$ when your evidence is $E^{\prime}$ survives learning the true epistemic value of the evidence $E$.

${ }^{51}$ Thanks to Paul Boghossian for discussion on this point.
} 
Still, our beliefs and credences play a serious role in our cognitive lives; beliefs in particular embody how we take the world to be. White and Kelly both consider whether permissivism requires "a departure from very natural ways of thinking about evidence and rationality." ${ }^{22}$ It may be that in order to reason, and in order to properly embrace the conclusions of reasoning, we must take that reasoning to have a kind of authority that is possible only if it is uniquely correct. ${ }^{53}$ There's a deepseated tension in permissivism between rational respect and normative authority; perhaps that tension supplies the best motivation for the Uniqueness Thesis. ${ }^{54}$

\section{REFERENCES}

Adams, W. J., E. W. Graf, and M. O. Ernst (2004). Experience can change the 'light-from-above' prior. Nature Neuroscience 7, 1057-1058.

Ballantyne, N. (2012). The problem of historical variability. In D. Machuca (Ed.), Disagreement and Skepticism, Routledge Studies in Contemporary Philosophy, pp. 239-259. Routledge.

Ballantyne, N. (2015). The significance of unpossessed evidence. The Philosophical Quarterly 65, 315-35.

Ballantyne, N. and E. Coffman (2011). Uniqueness, evidence, and rationality. Philosophers' Imprint 11, 1-13.

Ballantyne, N. and E. Coffman (2012). Conciliationism and uniqueness. Australasian Journal of Philosophy 90, 657-670.

Carnap, R. (1950). Logical Foundations of Probability. Chicago: University of Chicago Press.

Chalmers, D. (2007). The Matrix as metaphysics. In T. Gendler, S. Siegel, and T. Cahn (Eds.), The Elements of Philosophy. McGraw-Hill.

Christensen, D. (2009). Disagreement as evidence: The epistemology of controversy. Philosophy Compass 4, 756-767.

Christensen, D. (2016). Conciliation, uniqueness, and rational toxicity. Noûs 50, 584-603.

Cohen, S. (2013). A defense of the (almost) equal weight view. In J. Lackey and D. Christensen (Eds.), The Epistemology of Disagreement: New Essays, pp. 98-120. Oxford: Oxford University Press.

\footnotetext{
${ }^{52}$ The phrase is from (White 2014, p. 315); Kelly discusses it at his (2014, p. 309).

${ }^{53}$ Compare David Enoch's argument that realism about normative facts is indispensable for rational deliberation. (Enoch 2011, Ch. 3). Something like the tension we're pointing to may also be at play in Robert Mark Simpson's "arbitrariness objection" to permissivism (Simpson ta) and Jonathan Weisberg's "Instability Problem" (Weisberg ta).

${ }^{54}$ Thanks to Jochen Briesen, Rachael Briggs, Stewart Cohen, Juan Comesaña, Maria LasonenAarnio, Sarah Moss, Clinton Packman, Baron Reed, Darrell Rowbottom, Elliott Sober, Peter Vranas, Roger White, and a number of anonymous referees for comments on earlier versions of this material; to audiences at the Australian National University, the final Bellingham Summer Philosophy Conference, the Konstanz Reasoning Conference, the University of Bristol, the University of Michigan-Ann Arbor, Northwestern University's Sawyer Seminar in Social Epistemology, the University of Colorado-Boulder, and the 2010 meeting of the American Philosophical Association's Pacific Division; and to the participants in Titelbaum's spring 2011 seminar at the University of Wisconsin-Madison on the Objectivity of Reasons. Titelbaum's work on this article was supported by a Vilas Associates Award from the University of Wisconsin-Madison and a Visiting Fellowship from the Austrlian National University.
} 
Comesaña, J. (2014). Reply to pryor. In M. Steup, J. Turri, and E. Sosa (Eds.), Contemporary Debates in Epistemology (2nd ed.)., pp. 239-243. Wiley Blackwell.

Conee, E. and R. Feldman (2004). Evidentialism. Oxford: Oxford University Press.

de Finetti, B. (1972). Probability, Induction, and Statistics: The Art of Guessing. London: John Wiley \& Sons.

Dogramaci, S. and S. Horowitz (2016). An argument for uniqueness about evidential support. Philosophical Issues 26, 130-147.

Earman, J. (1992). Bayes or Bust? A Critical Examination of Bayesian Confirmation Theory. Cambridge, MA: The MIT Press.

Easwaran, K., L. Fenton-Glynn, C. Hitchcock, and J. D. Velasco (2016). Updating on the credences of others: Disagreement, agreement, and synergy. Philosophers' Imprint 16, 1-39.

Elga, A. (ms). Lucky to be rational. Unpublished paper presented at the Bellingham Summer Philosophy Conference on June 6, 2008.

Enoch, D. (2011). Taking Morality Seriously: A Defense of Robust Realism. Oxford: Oxford University Press.

Feldman, R. (2007). Reasonable religious disagreements. In L. M. Antony (Ed.), Philosophers without Gods: Meditations on Atheism and the Secular Life. Oxford: Oxford University Press.

Hicks, D. J. (2015). Epistemological depth in a GM crops controversy. Studies in History and Philosophy of Biological and Biomedical Sciences 50, 1-12.

Kelly, T. (2005). The epistemic significance of disagreement. Oxford Studies in Epistemology 1, 167-196.

Kelly, T. (2008). Evidence. In E. N. Zalta (Ed.), The Stanford Encyclopedia of Philosophy (Fall 2008 ed.).

Kelly, T. (2010). Peer disagreement and higher-order evidence. In R. Feldman and T. A. Warfield (Eds.), Disagreement, pp. 111-174. Oxford University Press.

Kelly, T. (2014). Evidence can be permissive. In M. Steup, J. Turri, and E. Sosa (Eds.), Contemporary Debates in Epistemology (2nd ed.)., pp. 298-312. Wiley Blackwell.

Kopec, M. (ms). A pluralistic account of epistemic rationality. Unpublished manuscript.

Kopec, M. and M. G. Titelbaum (2016). The uniqueness thesis. Philosophy Compass 11, 189-200.

Kuhn, T. S. (1970). The Structure of Scientific Revolutions (2nd ed.). Chicago: University of Chicago Press.

Lewis, D. (1971). Immodest inductive methods. Philosophy of Science 38, 54-63. Meacham, C. J. G. (2014). Impermissive bayesianism. Erkenntnis 79, 1185-1217.

Podgorksi, A. (2016). Dyanimic permissivism. Philosophical Studies 173, 19231939.

Putnam, H. (1981). Reason, Truth, and History. Cambridge: Cambridge University Press.

Rosen, G. (2001). Nominalism, naturalism, philosophical relativism. Philosophical Perspectives 15, 69-91.

Savage, L. J. (1954). The Foundations of Statistics. New York: Wiley. 
Schechter, J. (ms). Luck, rationality, and explanation: A reply to Elga's 'lucky to be rational'. Unpublished manuscript.

Schoenfield, M. (2014). Permission to believe: Why permissivism is true and what it tells us about irrelevant influences on belief. Noûs 48, 193-218.

Sharadin, N. (2015). A partial defense of permissivism. Ratio 28(2).

Simpson, R. M. (ta). Permissivism and the arbitrariness objection. Episteme. Forthcoming. Published online first October 4, 2016.

Titelbaum, M. G. (2010). Not enough there there: Evidence, reasons, and language independence. Philosophical Perspectives 24, 477-528.

Titelbaum, M. G. (2015). Rationality's fixed point (or: In defense of right reason). In T. S. Gendler and J. Hawthorne (Eds.), Oxford Studies in Epistemology, Volume 5, pp. 253-94. Oxford University Press.

Titelbaum, M. G. and M. Kopec (ms). Plausible permissivism. Unpublished manuscript.

Vavova, K. (ta). Irrelevant influences. Forthcoming in Philosophy and Phenomenological Research.

Weisberg, J. (ta). Could've thought otherwise. Philosophers' Imprint. Forthcoming.

White, R. (2005). Epistemic permissiveness. Philosophical Perspectives 19, 445459.

White, R. (2010). You just believe that becuase.... Philosophical Perspectives 24, $573-615$.

White, R. (2014). Evidence cannot be permissive. In M. Steup, J. Turri, and E. Sosa (Eds.), Contemporary Debates in Epistemology (2nd ed.)., pp. 312323. Wiley Blackwell.

Williams, B. (1986). Ethics and the Limits of Philosophy. Cambridge, MA: Harvard University Press.

Wright, C. (1992). Truth and Objectivity. Cambridge, MA: Harvard University Press.

Wright, C. (2004). Warrant for nothing (and foundations for free)? Supplement to the Proceedings of the Aristotelian Society, 167-212. 